\title{
Depression Subtypes in Pediatric Inflammatory Bowel Disease
}

\author{
Eva M. Szigethy ${ }^{\dagger}$, Ada O. Youk $\ddagger$, David Benhayon ${ }^{\dagger}$, Diane L. Fairclough $\S$, Melissa C. \\ Newara $^{\dagger}$, Margaret A. Kirshner ${ }^{\dagger}$, Simona I. Bujoreanull, Christine Mrakotsky ${ }^{\text {, Athos }}$ \\ Bousvaros ", Arvind I. Srinath ${ }^{*}$, David J. Keljo*, David J. Kupfer ${ }^{\dagger}$, and David R. DeMaso"I \\ *Department of Pediatric Gastroenterology, Children's Hospital of Pittsburgh, Pittsburgh, PA \\ †Department of Psychiatry, University of Pittsburgh School of Medicine, Pittsburgh, PA \\ ‡Department of Biostatistics, University of Pittsburgh, Pittsburgh, PA \\ §Department of Biostatistics and Informatics, Colorado School of Public Health, Aurora \\ "Department of Psychiatry, Boston Children's Hospital, Boston, MA \\ IDepartment of Pediatric Gastroenterology, Boston Children's Hospital, Boston, MA
}

\begin{abstract}
Objective-The association between inflammatory bowel disease (IBD) and depression provides a unique opportunity to understand the relation between systemic inflammation and depressive symptom profiles.
\end{abstract}

Methods-Youth $(\mathrm{n}=226)$ ages 9 to 17 years with comorbid IBD and depression underwent psychiatric assessment and evaluation of IBD activity. Latent profile analysis (LPA) identified depressive subgroups based on similar responses to the Children's Depression Rating ScaleRevised. Demographic factors, depression severity, anxiety, IBD activity, inflammatory markers, IBD-related medications, and illness perception were evaluated as predictors of profile membership.

Results-Mean age was 14.3 years; $75 \%$ had Crohn disease; $31 \%$ were taking systemic corticosteroids. Mean depressive severity was moderate, whereas IBD activity, which reflects inflammation, was mild. LPA identified 3 subgroups: Profile-1 (mild, 75\%) had diverse low-grade depressive symptoms and highest quality of life; Profile-2 (somatic, 19\%) had severe fatigue, appetite change, anhedonia, decreased motor activity, and depressed mood with concurrent highdose steroid therapy and the highest IBD activity; and Profile-3 (cognitive, 6\%) had the highest rates of self-reported depressive symptoms, ostomy placements, and anxiety with IBD symptoms in the relative absence of inflammation.

Conclusions-Evidence was found for 3 depression profiles in youth with IBD and depression. Our analyses determined that patients with predominantly somatic or cognitive symptoms of

(C) 2014 by European Society for Pediatric Gastroenterology, Hepatology, and Nutrition and North American Society for Pediatric Gastroenterology, Hepatology, and Nutrition

Address correspondence and reprint requests to Eva Szigethy, MD, PhD, Children's Hospital of Pittsburgh, 4401 Penn Avenue, Pittsburgh, PA 15224 (szigethye@upmc.edu).

The authors report no conflicts of interest. 
depression comprised $25 \%$ of our cohort. These findings may be used to design subgroup-specific interventions for depression in adolescents with IBD and other physical illnesses associated with systemic inflammation.

\section{Keywords}

comorbid psychiatric and physical illness; depression; inflammatory bowel disease; latent profile analysis; pediatric

Clinical depression is a heterogeneous disorder marked by the presence of different clusters of depressive symptoms. Not only does the symptom profile vary between individuals but different symptom clusters likely stem from different patho-physiological etiologies (1). One pathway to depression for which there is growing evidence involves the response of the central nervous system to systemic inflammation.

The studies on adult population support a link between depression and inflammation. Elevated inflammatory markers (eg, C-reactive protein [CRP], cytokines) have been associated with depression compared with that in healthy controls and have been linked to somatic and/or neurovegetative depressive symptoms (2-5). Patients with depression may exhibit activation of their immune system akin to the response to an infection (6). Increased systemic inflammation is prominent in physical illnesses, which are also associated with depression (eg, cardiovascular disease, cancer, multiple sclerosis) (7-11). The administration of exogenous cytokines (eg, interferon- $\alpha$ ) for hepatitis $\mathrm{C}$ and certain cancers has been associated with increased rates of depression (12-15). Although pretreatment with antidepressants may have a positive effect (16), differential treatment response of somatic versus cognitive symptoms suggests different underlying etiological pathways (12).

Collectively, these studies support causality between systemic inflammation and a subset of depressive symptoms and suggest the potential for novel anti-inflammatory therapies for depression (17).

In children and adolescents, the relation between inflammation and depression is less clear. One study demonstrated a link between endogenous depression and systemic inflammation, although only in adolescents with a history of childhood adversity (18). Several studies have reported an association between depression and worsened disease course in asthma and diabetes mellitus, 2 chronic childhood illnesses marked by systemic inflammation (19). Inflammatory bowel disease (IBD) is another chronic pediatric physical illness with significant immune system involvement and an association with depression $(20,21)$. IBD, consisting of Crohn disease (CD) and ulcerative colitis (UC), is characterized by lifelong recurrent bouts of gastrointestinal symptoms (eg, bloody diarrhea, intense pain) and medical therapies with profound adverse effects (22).

An association between somatic depressive symptoms and IBD activity in adolescents has been shown using a factor analysis of the Children's Depression Inventory (CDI) (23).

Although this analysis focused on how specific CDI items covary across patients, it did not investigate whether different clusters of depressive symptoms exist in adolescents with IBD. 
In the present study, a person-centered analysis is used to identify homogeneous groups of youth with IBD who have similar patterns of depressive symptoms and to assess uniquely associated demographic and clinical features, including IBD activity indices, which reflect disease-related inflammation. Although depressive symptoms were the primary focus, the severity of comorbid anxiety $(24,25)$, demographic factors $(18,26)$, and negative physical illness perception (27) were analyzed as predictors of the group membership. We hypothesized that patients with higher levels of somatic depressive symptoms have higher IBD activity (and related systemic inflammation), whereas those with higher levels of cognitive depressive symptoms would have increased psycho-pathology unrelated to IBD activity.

\section{METHODS}

This investigation was part of a large-scale randomized treatment trial at Children's Hospital of Pittsburgh and Boston Children's Hospital comparing cognitive behavioral therapy to supportive nondirective therapy treating depression. This study was approved by both hospital institutional review boards and conducted in accordance with institutional guidelines. Parents provided informed consent and adolescents provided assent.

\section{Participants}

In the trial, patients ages 9 to 17 years with biopsy-confirmed IBD were screened for depression with the CDI (28) during an outpatient visit to their gastroenterologist or while hospitalized with an IBD flare. As CDI scores $\geq 10$ on either child or parent rating (CDI-C/ CDI-P) have been found to be associated with clinically significant depressive symptoms (29), this cutoff was used to determine who would be interviewed using the Kiddie Schedule for Affective Disorders and Schizophrenia for Children, Present Version (KSADS-PL) (30). Youth were included in the trial if they met criteria for major or minor depression by the Diagnostic and Statistical Manual of Mental Disorders, Fourth Edition, Text Revision (DSM-IV-TR) criteria on the KSADS-PL (31). Minor depression is functionally impairing and has been shown to evolve to major depression, if left untreated (32). The KSADS-PL interviews occurred within 2 weeks of screening and were conducted either in outpatient clinics or over the telephone.

Exclusion criteria for the trial included the following: history or current episode of bipolar disorder, eating disorder, or psychotic disorder by DSM-IV-TR criteria using KSADS-PL; recent suicide attempt (within 1 month of study entry) or depressive severity requiring acute psychiatric hospitalization within 3 months of study entry; antidepressant medications within 1 month of assessment; substance abuse by history or iatrogenic opiate use within 1 month of study entry; and current treatment with psychotherapy.

\section{Measures}

The CDI (28) is a 27-item self-report questionnaire that assesses the frequency and severity of depressive symptoms. The CDI has well-validated psychometric properties in various developmental and ethnic groups and has been used to reliably diagnose depression in medically ill patients, including those with IBD (33). 
The Children's Depression Rating Scale-Revised (CDRS-R) is a validated semistructured clinician-rated scale consisting of 17 items (34). Total raw scores are based on the information collected from interviewing both the child and parent. The CDRS-R has acceptable internal consistency and test-retest reliability and was the primary outcome measure of depression (35). The KSADS-PL is a semistructured interview designed to ascertain present episode of psychiatric illness according to DSM-IV-TR criteria (30).

The Screen for Child Anxiety Related Emotional Disorders (SCARED) is a 41-item selfreport questionnaire using a 3-point scale designed to screen for anxiety severity in youth ages 9 to 18 years (36). The SCARED is completed by the parent and child independently and conforms to DSM-IV disorders. The scale has been validated in clinical and community samples with good internal consistency (37). A child-reported SCARED score $\geq 25$ is consistent with clinically significant anxiety (38).

The IMPACT III questionnaire is a validated self-report measure of health-related quality of life (HRQOL) designed for youth with IBD (39). It consists of 35 Likert-scaled questions measuring 6 quality-of-life domains (bowel symptoms, extra-intestinal symptoms, emotional functioning, social functioning, body image, and treatment/interventions). The IMPACT III has been shown to have good convergent validity and test-retest reliability for study participants ages 9 to 18 years (39).

The Pediatric Crohn's Disease Activity Index (PCDAI) is a well-validated scale used to determine present CD activity (40). The PCDAI includes measures in 3 illness domains that are routinely collected as part of the medical visit: self-reported abdominal pain, diarrhea, and functional disability; clinician-rated disease activity from examination; and laboratory measurements, including erythrocyte sedimentation rate (ESR), hematocrit, albumin, and growth parameters. The Pediatric Ulcerative Colitis Activity Index (PUCAI) is a validated symptom-based score used for UC activity (41).

Both the PCDAI and PUCAI were determined by a blinded study gastroenterologist based on the information from the medical record. Both indices were completed within 4 weeks of the depression screening. Because the PCDAI and PUCAI yield different indices, they were converted to standardized $z$ scores and combined into 1 activity measure. Plasma CRP was also included as inflammatory biomarker.

The Abdominal Pain Index (API) is a 5-item measure designed to characterize self-reported duration, severity, and frequency of pain experienced by the patient during the past 2 weeks (42). Scores $>15$ are indicative of clinically significant pain. Total scores were converted to $z$ scores for statistical analyses.

The Brief Illness Perception Questionnaire (BIPQ) is a 9-item validated scale assessing a patient's perception of overall effect of illness (43), including length of illness, possibility of control over illness, severity of physical symptoms, level of personal concern, understanding of illness, and emotional effect of illness. These items are scored on a continuous 0 to 10 scale, with higher scores associated with more negative illness perception. 
The general information sheet was used to obtain demographic information (eg, age, race, sex, socioeconomic status [SES]) using an occupation-based measure, the Revised Duncan (44). The SES was defined as stratified household income divided by the number of individuals in the household.

Medical history including onset, presentation, and course of IBD; location of disease in the gastrointestinal tract; and medication use (eg, steroids, biologics, immunomodulators) was obtained from parent reports and verified in the medical record by a blinded gastroenterologist. Surgical history and present ostomy status were recorded. IBD course was divided into the following categories: acute (diagnosis $\$ 6$ months; chronic diagnosis $>6$ months with $<1$ month in remission defined by inactive score on disease severity measures [PCDAI/PUCAI]); and chronic intermittent (diagnosis $>6$ months with at least 1 month of remission).

\section{Statistical Analysis}

Latent profile analysis (LPA) was used to identify distinct subgroups that shared similar symptoms (profiles) from the 17 items of the CDRS-R. LPA is a multivariate modeling technique for identifying latent (unmeasured) groups of individuals within a population using continuous measurement variables. Mplus (version 5.1; Muthen \& Muthen, Los Angeles, CA) was used to determine the best-fitting model from a set of models ranging from 1 to 5 profiles. Criteria used to select the best model included the Lo-Mendell-Rubin (LMR) adjusted likelihood ratio test, which compares an $n$ profile model to an $n-1$ profile model; the Bayes information criteria (BIC) to examine goodness of fit; and entropy, which gives a measure of classification (a value of 1 indicates perfect classification).

The final model was selected based on an LMR statistic that was statistically significant ( $\mathrm{n}$ profiles were better than $n-1$ profiles), a low Bayes information criteria value (compared with the other profile models), and an entropy value close to 1 . Clinical interpretation of the groups was included in the selection of the best number of profiles. After determining profile membership, we used univariate multinomial logistic regression models to determine whether demographic or clinical covariates were predictive of profile membership. Owing to the highly skewed nature of the ESR and CRP distributions, these variables were logtransformed for analysis. Regression analyses were performed using STATA (version 12.0; StataCorp, College Station, TX). No attempts were made to adjust for multiple comparisons because this study was hypothesis generating in nature.

\section{RESULTS}

\section{Sample Characteristics}

A total of 765 subjects were screened during a 3-year period, with 226 meeting DSM-IV-TR criteria for major depression or minor depression. Of the 539 excluded subjects, $433 \mathrm{did}$ not meet depressive threshold criteria, 47 were not interested, and the rest met at least 1 of the exclusion criteria. The total sample was predominantly female, mostly white, and had a mean age of 14.3 years (standard deviation 2.42) (Table 1). There was no significant difference between the depressed and nondepressed groups with regard to age and sex. In 
terms of IBD activity (using combined $z$ scores for UC and CD), there was significantly greater IBD activity in the depressed $(0.49)$ versus nondepressed $(-0.24)$ group $(P<0.0001)$.

Both depression scores (CDRS-R and CDI) were consistent with moderate depressive severity. Comorbid DSM-IV-TR psychiatric diagnoses are summarized in Table 2. Seventyfive percent of the total sample had CD with overall mild-to-moderate IBD activity. Thirtythree percent of subjects had newly diagnosed IBD, and $28.3 \%$ had a chronic IBD course. Twenty-five percent of the sample was taking high-dose ( $\geq 20 \mathrm{mg} /$ day) systemic steroids, $31 \%$ of the sample was taking any amount of steroids, $21.2 \%$ taking biologics alone or biologics with immunomodulators, $31.0 \%$ taking immunomodulators without steroids or biologics, and $9.9 \%$ required surgery, with $4.0 \%$ requiring an ostomy (Table 1 ).

\section{Subtypes of Depression}

Three profiles emerged as the best fit and had the most meaningful clinical interpretation. The comparison of 3 to 2 profiles was statistically significant, suggesting that the 3-profile model was preferable to the 2-profile model (LMR, $P=0.002$ ). Because the 4-profile model did not statistically differ from the 3-profile model (LMR, $P=0.476$ ), the 3-profile model was chosen. In addition, the entropy value for the 3-profile model was close to 1 (entropy = $0.956)$, and the gain in entropy from 3 to 4 profiles was minimal $(0.01)$.

Approximately $75 \%$ of the participants were in Profile-1, 19.5\% in Profile-2, and 5.7\% in Profile-3 (Fig. 1). These depression profiles were defined as mild (Profile-1), disease related or somatic (Profile-2), and cognitive despair (Profile-3) based on the following criteria:

1. Profile-1 (mild depression) had lower depressive symptom ratings across all 17 items, with its highest mean scores being fatigue (4.1), irritability (4.0), and depressed feelings (3.5).

2. Profile-2 (somatic depression) had the highest ratings for anhedonia (mean 4.7), change in appetite (mean 4.1), fatigue (mean 6.0), physical complaints (mean 5.0), irritability (mean 4.3), and depressed feelings (mean 5.0). This profile also had the highest ratings on observed depressive symptoms of facial affect (mean 3.8), listless speech (mean 2.4), and decreased motor activity (mean 3.2). This profile differed most from the other 2 profiles in symptoms of depressed affect and motor hypoactivity.

3. Profile-3 (cognitive despair) rated highest on morbid ideation (mean 2.8), suicidal ideation (mean 3.5), and weeping (mean 4.2). This profile demonstrated high mean scores for fatigue (5.6) and depressed feelings (4.4). This profile differed most from Profile-1 and Profile-2 on hopelessness and suicidal ideation.

Table 3 shows the results of multinomial logistic regression models, demonstrating association with demographic and clinical variables by depressive profile. For Profile-2, concurrent systemic high-dose steroid therapy significantly predicted profile membership compared with Profile-1. Profile-2 differentiated from Profile-1 but not Profile-3 in having more severe clinician-rated depressive severity (CDRS-R), poorer HRQOL, increased IBD activity, ostomies, and greater use of immunomodulators. IBD activity was significantly higher in both Profile-2 and Profile-3 compared to Profile-1, as well as in Profile-2 
compared with Profile-3. In addition, the highest levels of log ESR and log CRP and the highest level of both child- and parent-rated abdominal pain were present in Profile-2.

To evaluate the potential confounding effects of anti-inflammatory medications (specifically steroids, immunomodulators, and biologics) on the relation between inflammation and depression, these medication variables were added individually into prediction models. For inflammation $(\log$ ESR), the statistical significance $(P=0.028)$ between Profile- 2 and Profile-1 was reduced after controlling for high-dose steroids $(P=0.110)$ or for immunomodulators $(P=0.056)$, but not for biologics or a combination of both biologics and immunomodulators. For CD activity, statistical significance for Profile-2 versus Profile-1 ( $P$ $<0.0001$ ) was retained after controlling for each medication category.

For Profile-3 (cognitive despair), depressive severity and longer duration since IBD diagnosis significantly predicted profile membership. Profile-3 had higher child-rated anxiety levels (SCARED-C) compared with both Profile-1 $(P=0.110)$ and Profile-2 $(P=$ $0.087)$ with marginal statistical significance. Although the groups did not differentiate in terms of frequency of surgery, Profile-3 had the most members with ostomies.

The 3 profiles did not differentiate from each other in terms of age, sex, SES, or ethnicity; however, patients in Profile-2 were predominantly girls (56.8\%) and had lower SES compared with the other 2 groups, although not statistically significant. Ninety-two percent (12/13) of patients in Profile-3 had CD.

\section{DISCUSSION}

This is the first investigation to assess the coaggregation of depressive symptoms in a pediatric IBD population. Three distinct depressive symptom profiles emerged, each with clinical correlates. Profile-1 (mild depression), the largest group, was marked by the presence of low-grade depressive symptoms. Profile-2 (somatic depression) exhibited disease-related depressive symptoms, and Profile-3 (cognitive despair), the smallest group, was characterized by depressive symptoms of cognitive despair. The validity of the 3 profiles was supported by distinct patterns of association with clinical variables, particularly Profile-2 and Profile-3, which together represented $25 \%$ of the sample, a sizable proportion in a multidetermined psychiatric disorder.

Profile-2 consisted of individuals with higher scores on somatic depressive items and clinician-observed depressive symptoms. This symptom cluster has been reported in other cohorts with inflammation-related depression $(5,45,46)$ and in depressed behaviors observed in animal models of cytokine-mediated sickness behavior $(47,48)$. Profile- 2 had the highest IBD activity and systemic inflammation, consistent with previous reports $(21,49,50)$. The direction of the relation is consistent with a depressive phenotype defined by the effects of systemic inflammation on the brain. Future studies could examine other inflammatory markers or cytokines such as tumor necrosis factor (TNF)- $\alpha$, interleukin-1 $\beta$, and interleukin-6, which have been elevated in adults with depression (2-4).

The finding that greater abdominal pain predicts membership in this group with high inflammatory activity is consistent with previous work showing that $50 \%$ to $70 \%$ of patients 
with IBD have abdominal pain during disease flares (51) and direct correlation between rectal hypersensitivity and inflammation (52); however, it is also possible that pain is occurring as a result of somatization or visceral hypersensitivity that has been reported in IBD cohorts in the absence of disease activity $(53,54)$.

Interestingly, treatment with biologics (TNF-a antagonists) did not differentiate the 3 profiles, suggesting that systemic treatment of inflammation is not sufficient to alter brainassociated changes. This finding is consistent with a recent trial finding that the TNF-a antagonist, infliximab, was not efficacious for adults with treatment refractory depression (55). It is important to note that controlling for other anti-inflammatory medications such as steroids or immunomodulators did influence the relation between depression and inflammation (as measured by ESR), although not IBD activity. This finding suggests further study is needed to understand how to develop better psychiatric interventions, targeting the neuropsychiatric manifestations of IBD.

Although not reaching statistical significance, female predominance seen in Profile-2 is consistent with findings in adolescents with endogenous depression, in which somatic symptoms such as changes in appetite, sleep, and psychomotor retardation are more common in girls (56). Subjects in Profile-2 also had the lowest socioeconomic status, consistent with studies showing that childhood adversity is a risk factor for the development of inflammatory depression $(18,57)$. Collectively, these findings warrant further investigation of the manner in which cytokines may mediate brain changes underlying somatic depressive symptoms in larger samples.

Membership in Profile-2 was significantly predicted by a concurrent high-dose steroid therapy. Steroid use has been associated with irritability, manic activation, insomnia, and cognitive symptoms in other studies of patients with pediatric and adult IBD (58-60). To evaluate whether steroid use altered the clustering of depressive symptoms, LPA was completed excluding participants on high-dose steroids but found the profile structure did not change. These findings suggest that being on high-dose systemic steroids may serve as a proxy for elevated IBD activity and not a unique steroid-induced depressive phenotype (59).

Profile-3 endorsed hopelessness, suicidal ideation, and weeping, all consistent with cognitive despair. This profile was characterized by more severe self-reported depressive symptoms, highest frequency of ostomies, and longest duration of IBD compared with the other 2 groups. Profile-3 had elevated disease activity and pain ratings in the relative absence of inflammation, suggesting that depression may predispose to exaggerated negative perceptions of somatic stimuli.

Although anxiety symptoms did not significantly predict group membership, Profile- 3 was the only group with a clinically significant anxiety severity rating. This finding is consistent with adults with IBD, wherein there is a relation among pain, anxiety, and negative cognitive distortions (53). The presence of anxiety and moderate fatigue in Profile-3 is consistent with a report that chronic fatigue is associated with increased IBD-related worries in adults (61). High levels of anxiety, including IBD-related worries, have been reported in both adolescents and adults with IBD $(62,63)$. Therefore, larger-scale studies would help 
determine whether different domains of anxiety (eg, somatic symptoms versus separation versus school avoidance) map onto different depressive symptom clusters. Alternatively, it is possible that greater depressive severity driven by more cognitive depressive symptoms is independent of IBD-related factors and is related to other predisposing risk factors (eg, pubertal delay, early-life trauma, genetic polymorphisms) not measured in this study.

Although the cross-sectional nature of this study prevents claims of causality, the findings are consistent with the existence of distinct depressive subtypes differing in both symptom presentation and clinical correlates. Thus, youth who are experiencing active inflammation or those with more chronic and longstanding IBD may benefit from screening for depression and intensive psychosocial intervention. Our treatment study of adolescents with IBD and subsyndromal depression demonstrated that somatic depressive symptoms had greater improvement with a combination of medical treatment and adjunctive cognitive behavioral therapy as compared to medical treatment alone (64).

Profile-1 showed a broader range of depressive items, with individual scores being lower than the other 2 groups. This profile reported the highest HRQOL and relatively mild disease activity, although the presence of steroid use in $18 \%$ of this subsample suggests some heterogeneity in IBD activity status. It remains challenging to separate the psychosocial effect of having a lifelong diagnosis from disease-related depression. For example, fatigue, the most highly rated symptom in this profile, is multidetermined and can have physical and/or psychological causes. It is also possible that other potential predisposing depressive factors independent of IBD are involved such as genetic vulnerability, metabolic factors, obesity, psychosocial stressors, or comorbid psychiatric diagnoses $(14,65,66)$. Understanding the neurobiological mechanisms underlying different clusters of depressive symptoms may lead to better individualized treatment strategies and help decrease related problems such as poor medical adherence and poor quality of life.

The manner in which depression is defined may have influenced the LPA. Differences can occur based on informant source, instruments used, and diagnostic criteria (eg, atypical vs melancholic depression) (1). Some instruments exclude somatic depressive items such as fatigue, sleep, and appetite altogether because they have been linked more to severity of physical illness rather than depressive status. These symptoms were not excluded from the present study because at least in some patients, they were present in the relative absence of IBD activity.

Clinically significant depression can be defined using existing nosological frameworks such as the DSM-IV-TR or based upon cutoffs of existing scales. In the present study, we used the categorical criteria defined by the DSM-IV-TR for enrollment, and the dimensional CDRS-R was used as an age-appropriate validated scale to include both somatic and nonsomatic depressive symptoms, even though it likely underrepresents aspects of atypical depression, such as hypersomnia and increased appetite (67), which occur frequently in subjects with IBD.

The relatively small sample size $(\mathrm{n}=226)$ for a subject-centered analysis evaluating subtypes of depression, particularly the small number of subjects $(n=13)$ in Profile- 3 , is a 
limitation. Another limitation is the relative sample heterogeneity with regard to IBD phenotypes, site differences, and differences in psychiatric comorbidities. Nevertheless, this exploratory study yields important constructs worth pursuing to better identify depressive phenotypes, which may benefit from different treatment approaches. Larger multisite studies will be necessary to evaluate if different IBD phenotypes have a unique relation with different depressive subtypes and/or other psychopathological subtypes. Future work will include assessing the stability of these latent depressive profiles over time.

\section{CONCLUSIONS}

This investigation is an innovative first step in characterizing depressive subtypes in youth with a chronic systemic inflammatory condition. One-fourth of youth with cooccurring IBD and depression were characterized by either disabling somatic symptoms or cognitive despair. The study provides an important framework for fine-tuning the early characterization of pediatric depressive symptoms in IBD. The investigation of the 3 latent profiles identified in these analyses longitudinally (ie, at various time points within the same patient) could help differentiate whether depressive symptoms are primarily associated with systemic inflammation versus a cognitive reaction to a long-term illness. Furthermore, such exploration across different chronic disease samples will provide a richer picture of adolescent depression that can inform nosological and etiological advances, leading to early identification and preventive interventions.

\section{Acknowledgments}

This research was funded by the National Institute of Mental Health (R01 MH077770) and the NIH Director's Innovator Award (1DP2OD001210).

\section{References}

1. Lamers F, Burstein M, He JP, et al. Structure of major depressive disorder in adolescents and adults in the US general population. Br J Psychiatry. 2012; 201:143-50. [PubMed: 22700082]

2. Alesci S, Martinez PE, Kelkar S, et al. Major depression is associated with significant diurnal elevations in plasma interleukin-6 levels, a shift of its circadian rhythm, and loss of physiological complexity in its secretion: clinical implications. J Clin Endocrinol Metab. 2005; 90:2522-30. [PubMed: 15705924]

3. Dowlati Y, Herrmann N, Swardfager W, et al. A meta-analysis of cytokines in major depression. Biol Psychiatry. 2010; 67:446-57. [PubMed: 20015486]

4. Howren MB, Lamkin DM, Suls J. Associations of depression with C-reactive protein, IL-1, and IL-6: a meta-analysis. Psychosom Med. 2009; 71:171-86. [PubMed: 19188531]

5. Raison CL, Capuron L, Miller AH. Cytokines sing the blues: inflammation and the pathogenesis of depression. Trends Immunol. 2006; 27:24-31. [PubMed: 16316783]

6. Miller AH, Maletic V, Raison CL. Inflammation and its discontents: the role of cytokines in the pathophysiology of major depression. Biol Psychiatry. 2009; 65:732-41. [PubMed: 19150053]

7. Gold SM, Irwin MR. Depression and immunity: inflammation and depressive symptoms in multiple sclerosis. Neurol Clin. 2006; 24:507-19. [PubMed: 16877121]

8. Jacobson CM, Rosenfeld B, Pessin H, et al. Depression and IL-6 blood plasma concentrations in advanced cancer patients. Psychosomatics. 2008; 49:64-6. [PubMed: 18212178]

9. Lutgendorf SK, Weinrib AZ, Penedo F, et al. Interleukin-6, cortisol, and depressive symptoms in ovarian cancer patients. J Clin Oncol. 2008; 26:4820-7. [PubMed: 18779606] 
10. Musselman DL, Miller AH, Porter MR, et al. Higher than normal plasma interleukin-6 concentrations in cancer patients with depression: preliminary findings. Am J Psychiatry. 2001; 158:1252-7. [PubMed: 11481159]

11. Miller GE, Freedland KE, Duntley S, et al. Relation of depressive symptoms to C-reactive protein and pathogen burden (cytomegalovirus, herpes simplex virus, Epstein-Barr virus) in patients with earlier acute coronary syndromes. Am J Cardiol. 2005; 95:317-21. [PubMed: 15670537]

12. Capuron L, Gumnick JF, Musselman DL, et al. Neurobehavioral effects of interferon-alpha in cancer patients: phenomenology and paroxetine responsiveness of symptom dimensions. Neuropsychopharmacology. 2002; 26:643-52. [PubMed: 11927189]

13. Capuron L, Hauser P, Hinze-Selch D, et al. Treatment of cytokine-induced depression. Brain Behav Immun. 2002; 16:575-80. [PubMed: 12401471]

14. Lotrich FE, El-Gabalawy H, Guenther LC, et al. The role of inflammation in the pathophysiology of depression: different treatments and their effects. J Rheumatol Suppl. 2011; 88:48-54. [PubMed: 22045979]

15. Valentine AD, Meyers CA, Kling MA, et al. Mood and cognitive side effects of interferon-alpha therapy. Semin Oncol. 1998; 25 (1 suppl 1):39-47. [PubMed: 9482539]

16. Musselman DL, Lawson DH, Gumnick JF, et al. Paroxetine for the prevention of depression induced by high-dose interferon alfa. N Engl J Med. 2001; 344:961-6. [PubMed: 11274622]

17. Lotrich F. Depression symptoms, low-grade inflammatory activity, and new targets for clinical intervention. Biol Psychiatry. 2011; 70:111-2. [PubMed: 21708303]

18. Miller GE, Cole SW. Clustering of depression and inflammation in adolescents previously exposed to childhood adversity. Biol Psychiatry. 2012; 72:34-40. [PubMed: 22494534]

19. Hood KK, Lawrence JM, Anderson A, et al. Metabolic and inflammatory links to depression in youth with diabetes. Diabetes Care. 2012; 35:2443-6. [PubMed: 23033243]

20. Greenley RN, Hommel KA, Nebel J, et al. A meta-analytic review of the psychosocial adjustment of youth with inflammatory bowel disease. J Pediatr Psychol. 2010; 35:857-69. [PubMed: 20123705]

21. Szigethy E, Levy-Warren A, Whitton S, et al. Depressive symptoms and inflammatory bowel disease in children and adolescents: a cross-sectional study. J Pediatr Gastroenterol Nutr. 2004; 39:395-403. [PubMed: 15448431]

22. Regueiro, MD.; Swoger, JM. Clinical Challenges and Complications of IBD. Thorofare, NJ: SLACK Inc; 2013.

23. Thompson RD, Delaney P, Flores I, et al. Cognitive-behavioral therapy for children with comorbid physical illness. Child Adolesc Psychiatr Clin N Am. 2011; 20:329-48. [PubMed: 21440859]

24. Angold A, Costello EJ, Erkanli A. Comorbidity. J Child Psychol Psychiatry. 1999; 40:57-87. [PubMed: 10102726]

25. Ferdinand RF, de Nijs PF, van Lier P, et al. Latent class analysis of anxiety and depressive symptoms in referred adolescents. J Affect Disord. 2005; 88:299-306. [PubMed: 16182373]

26. Costello EJ, Angold A, Burns BJ, et al. The Great Smoky Mountains Study of Youth. Goals, design, methods, and the prevalence of DSM-III-R disorders. Arch Gen Psychiatry. 1996; 53:1129-36. [PubMed: 8956679]

27. McLafferty L, Craig A, Levine A, et al. Thematic analysis of physical illness perceptions in depressed youth with inflammatory bowel disease. Inflamm Bowel Dis. 2011; 17 (suppl 1):S54.

28. Kovacs, M. Children's Depression Inventory (CDI) Manual. New York: Multi-Health Systems; 1992.

29. Szigethy E, Kenney E, Carpenter J, et al. Cognitive-behavioral therapy for adolescents with inflammatory bowel disease and sub-syndromal depression. J Am Acad Child Adolesc Psychiatry. 2007; 46:1290-8. [PubMed: 17885570]

30. Kaufman J, Birmaher B, Brent D, et al. Schedule for Affective Disorders and Schizophrenia for School-Age Children-Present and Lifetime Version (K-SADS-PL): initial reliability and validity data. J Am Acad Child Adolesc Psychiatry. 1997; 36:980-8. [PubMed: 9204677]

31. Diagnostic and Statistical Manual of Mental Disorders. 4. Washington, DC: American Psychiatric Association; 2000. Text Revision 
32. Gonzalez-Tejera G, Canino G, Ramirez R, et al. Examining minor and major depression in adolescents. J Child Psychol Psychiatry. 2005; 46:888-99. [PubMed: 16033637]

33. Engstrom I. Mental health and psychological functioning in children and adolescents with inflammatory bowel disease: a comparison with children having other chronic illnesses and with healthy children. J Child Psychol Psychiatry. 1992; 33:563-82. [PubMed: 1577899]

34. Poznanski, EO.; Mokros, HB. Children's Depression Rating Scale, Revised (CDRS-R) Manual. Los Angeles: Western Psychological Services; 1996.

35. Poznanski EO, Grossman JA, Buchsbaum Y, et al. Preliminary studies of the reliability and validity of the children's depression rating scale. J Am Acad Child Psychiatry. 1984; 23:191-7. [PubMed: 6715741]

36. Birmaher B, Khetarpal S, Brent D, et al. The Screen for Child Anxiety Related Emotional Disorders (SCARED): scale construction and psychometric characteristics. J Am Acad Child Adolesc Psychiatry. 1997; 36:545-53. [PubMed: 9100430]

37. Pavuluri M, Birmaher B. A practical guide to using ratings of depression and anxiety in child psychiatric practice. Curr Psychiatry Rep. 2004; 6:108-16. [PubMed: 15038913]

38. Birmaher B, Brent DA, Chiappetta L, et al. Psychometric properties of the Screen for Child Anxiety Related Emotional Disorders (SCARED): a replication study. J Am Acad Child Adolesc Psychiatry. 1999; 38:1230-6. [PubMed: 10517055]

39. Otley A, Smith C, Nicholas D, et al. The IMPACT questionnaire: a valid measure of health-related quality of life in pediatric inflammatory bowel disease. J Pediatr Gastroenterol Nutr. 2002; 35:557-63. [PubMed: 12394384]

40. Hyams JS, Ferry GD, Mandel FS, et al. Development and validation of a Pediatric Crohn's Disease Activity Index. J Pediatr Gastroenterol Nutr. 1991; 12:439-47. [PubMed: 1678008]

41. Turner D, Otley AR, Mack D, et al. Development, validation, and evaluation of a pediatric ulcerative colitis activity index: a prospective multicenter study. Gastroenterology. 2007; 133:42332. [PubMed: 17681163]

42. Walker LS, Smith CA, Garber J, et al. Development and validation of the Pain Response Inventory for children. Psychol Assess. 1997; 9:392-405.

43. Broadbent E, Petrie KJ, Main J, et al. The brief illness perception questionnaire. J Psychosom Res. 2006; 60:631-7. [PubMed: 16731240]

44. Nakao, K.; Treas, J. The 1989 Socioeconomic Index of Occupations: Construction from the 1989 Occupational Prestige Scores (General Social Survey Methodological Report no. 74). Chicago, IL: University of Chicago; 1992.

45. Dantzer R, Kelley KW. Twenty years of research on cytokine-induced sickness behavior. Brain Behav Immun. 2007; 21:153-60. [PubMed: 17088043]

46. Pusztai L, Mendoza TR, Reuben JM, et al. Changes in plasma levels of inflammatory cytokines in response to paclitaxel chemotherapy. Cytokine. 2004; 25:94-102. [PubMed: 14698135]

47. Dantzer R, O'Connor JC, Freund GG, et al. From inflammation to sickness and depression: when the immune system subjugates the brain. Nat Rev Neurosci. 2008; 9:46-56. [PubMed: 18073775]

48. Pollak Y, Yirmiya R. Cytokine-induced changes in mood and behaviour: implications for 'depression due to a general medical condition', immunotherapy and antidepressive treatment. Int J Neuropsychopharmacol. 2002; 5:389-99. [PubMed: 12466037]

49. Hauser W, Schmidt C, Stallmach A. Depression and mucosal pro-inflammatory cytokines are associated in patients with ulcerative colitis and pouchitis—a pilot study. J Crohns Colitis. 2011; 5:350-3. [PubMed: 21683306]

50. Szigethy E, McLafferty L, Goyal A. Inflammatory bowel disease. Pediatr Clin North Am. 2011; 58:903-20. [PubMed: 21855713]

51. Wagtmans MJ, Verspaget HW, Lamers CB, et al. Crohn's disease in the elderly: a comparison with young adults. J Clin Gastroenterol. 1998; 27:129-33. [PubMed: 9754773]

52. Andersson $P$, Olaison G, Hallbook $O$, et al. Increased anal resting pressure and rectal sensitivity in Crohn's disease. Dis Colon Rectum. 2003; 46:1685-9. [PubMed: 14668596]

53. Farrokhyar F, Marshall J, Easterbrook B, et al. Functional gastrointestinal disorders and mood disorders in patients with inactive inflammatory bowel disease: prevalence and impact on health. Inflamm Bowel Dis. 2006; 12:38-46. [PubMed: 16374257] 
54. Minderhoud IM, Oldenburg B, Wismeijer JA, et al. IBS-like symptoms in patients with inflammatory bowel disease in remission; relationships with quality of life and coping behavior. Dig Dis Sci. 2004; 49:469-74. [PubMed: 15139501]

55. Raison CL, Rutherford RE, Woolwine BJ, et al. A randomized controlled trial of the tumor necrosis factor antagonist infliximab for treatment-resistant depression: the role of baseline inflammatory biomarkers. JAMA Psychiatry. 2013; 70:31-41. [PubMed: 22945416]

56. Bennett DS, Ambrosini PJ, Kudes D, et al. Gender differences in adolescent depression: do symptoms differ for boys and girls? J Affect Disord. 2005; 89:35-44. [PubMed: 16219362]

57. Danese A, Moffitt TE, Harrington H, et al. Adverse childhood experiences and adult risk factors for age-related disease: depression, inflammation, and clustering of metabolic risk markers. Arch Pediatr Adolesc Med. 2009; 163:1135-43. [PubMed: 19996051]

58. Mrakotsky C, Forbes PW, Bernstein JH, et al. Acute cognitive and behavioral effects of systemic corticosteroids in children treated for inflammatory bowel disease. J Int Neuropsychol Soc. 2013; 19:96-109. [PubMed: 23157730]

59. Suvak B, Beyazit Y, Sayilir A, et al. Effects of corticosteroid therapy on depression and inflammation in ulcerative colitis patients. J Crohns Colitis. 2011; 5:375-6. [PubMed: 21683313]

60. Fardet L, Kassar A, Cabane J, et al. Corticosteroid-induced adverse events in adults: frequency, screening and prevention. Drug Safety. 2007; 30:861-81. [PubMed: 17867724]

61. Jelsness-Jorgensen LP, Bernklev T, Henriksen M, et al. Chronic fatigue is associated with increased disease-related worries and concerns in inflammatory bowel disease. World $\mathbf{J}$ Gastroenterol. 2012; 18:445-52. [PubMed: 22346250]

62. Goodhand JR, Wahed M, Mawdsley JE, et al. Mood disorders in inflammatory bowel disease: relation to diagnosis, disease activity, perceived stress, and other factors. Inflamm Bowel Dis. 2012; 18:2301-9. [PubMed: 22359369]

63. Walker JR, Ediger JP, Graff LA, et al. The Manitoba IBD cohort study: a population-based study of the prevalence of lifetime and 12-month anxiety and mood disorders. Am J Gastroenterol. 2008; 103:1989-97. [PubMed: 18796096]

64. Szigethy E, Craig AE, Iobst EA, et al. Profile of depression in adolescents with inflammatory bowel disease: implications for treatment. Inflamm Bowel Dis. 2009; 15:69-74. [PubMed: 18831071]

65. Rao U, Chen LA. Characteristics, correlates, and outcomes of childhood and adolescent depressive disorders. Dialogues Clin Neurosci. 2009; 11:45-62. [PubMed: 19432387]

66. Shelton RC, Miller AH. Eating ourselves to death (and despair): the contribution of adiposity and inflammation to depression. Prog Neurobiol. 2010; 91:275-99. [PubMed: 20417247]

67. Paykel ES, Parker RR, Rowan PR, et al. Nosology of atypical depression. Psychol Med. 1983; 13:131-9. [PubMed: 6844458] 


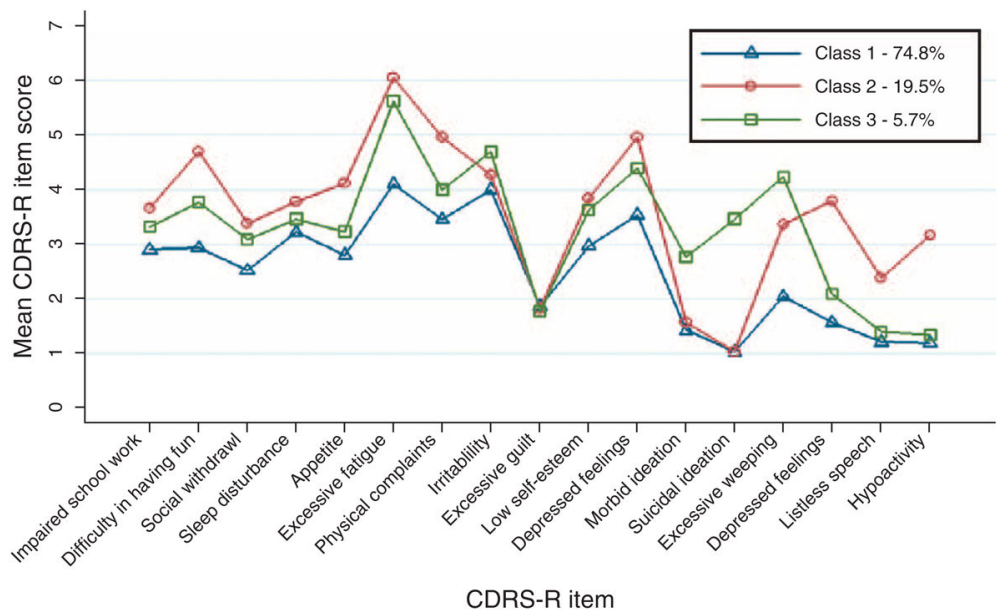

FIGURE 1.

Three distinct classes of youth estimated from a latent class model. 


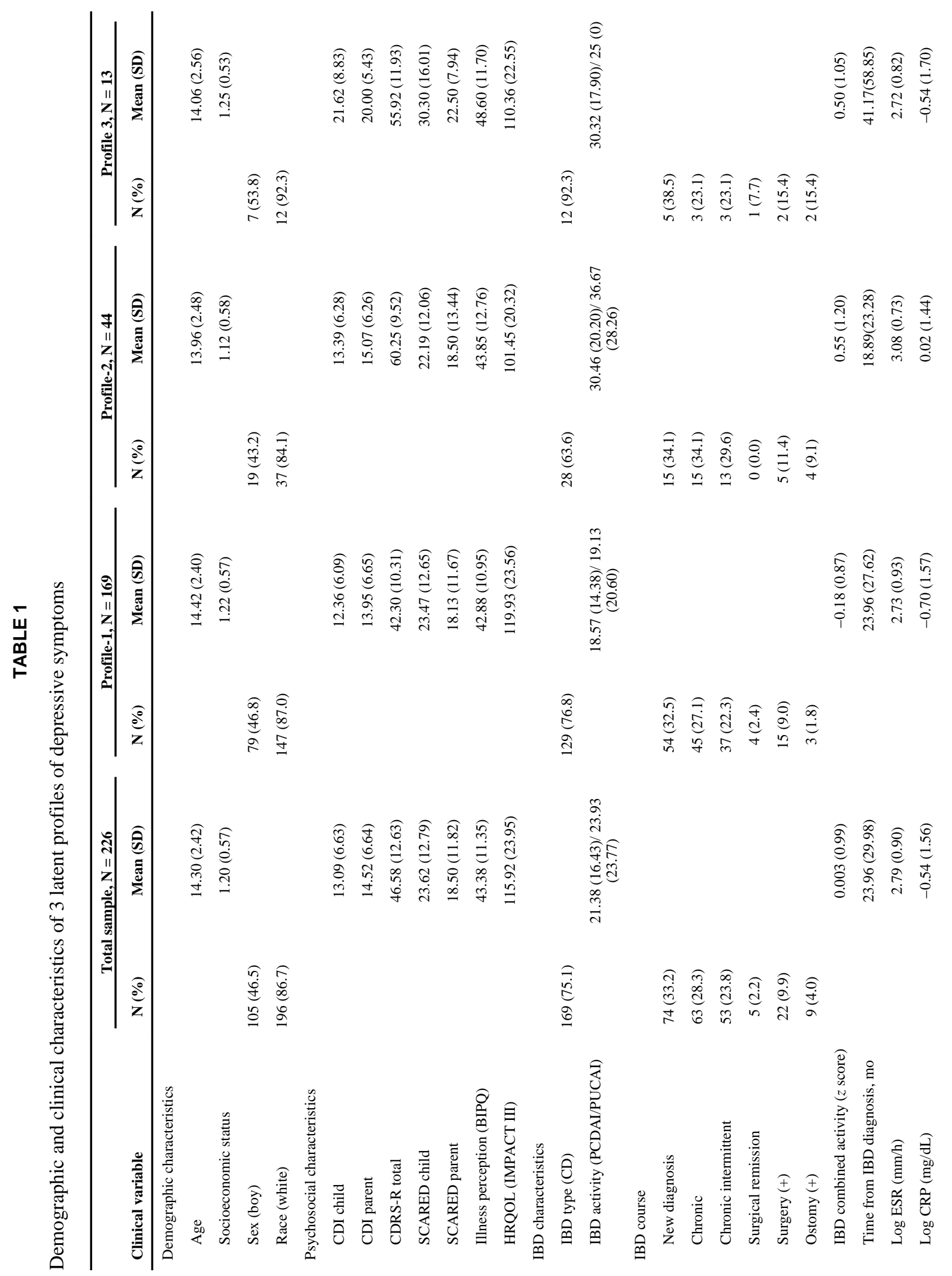


Szigethy et al.

Page 16

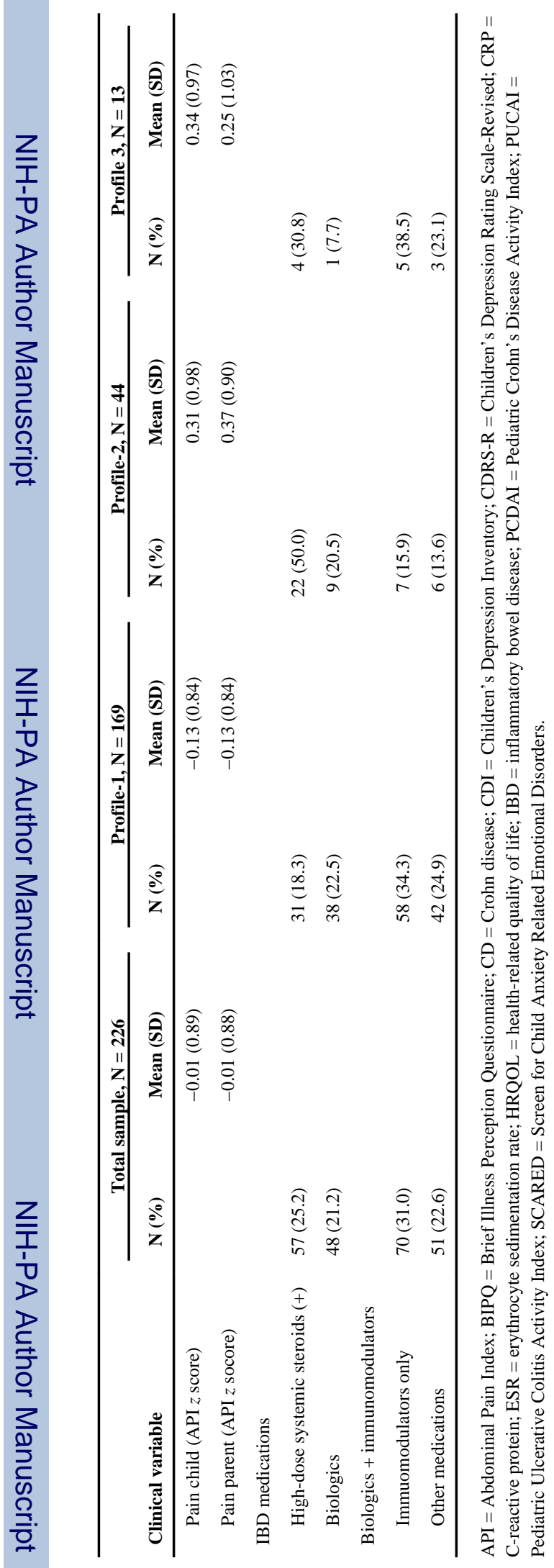

J Pediatr Gastroenterol Nutr. Author manuscript; available in PMC 2014 July 29. 
TABLE 2

Frequency of comorbid psychiatric diagnoses in depressed youth with IBD $(n=226)$

\begin{tabular}{lr}
\hline Psychiatric diagnoses & Frequency, n \\
\hline Generalized anxiety disorder & 47 \\
Specific phobia & 34 \\
Attention-deficit/hyperactivity disorder & 25 \\
Separation anxiety disorder & 21 \\
Oppositional defiant disorder & 20 \\
Social anxiety & 19 \\
Obsessive compulsive disorder & 4 \\
Dysthymia & 3 \\
Panic disorder & 3 \\
Posttraumatic stress disorder & 3 \\
Anorexia nervosa & 2 \\
Conduct disorder & 1 \\
\hline
\end{tabular}

$\mathrm{IBD}=$ inflammatory bowel disease. 


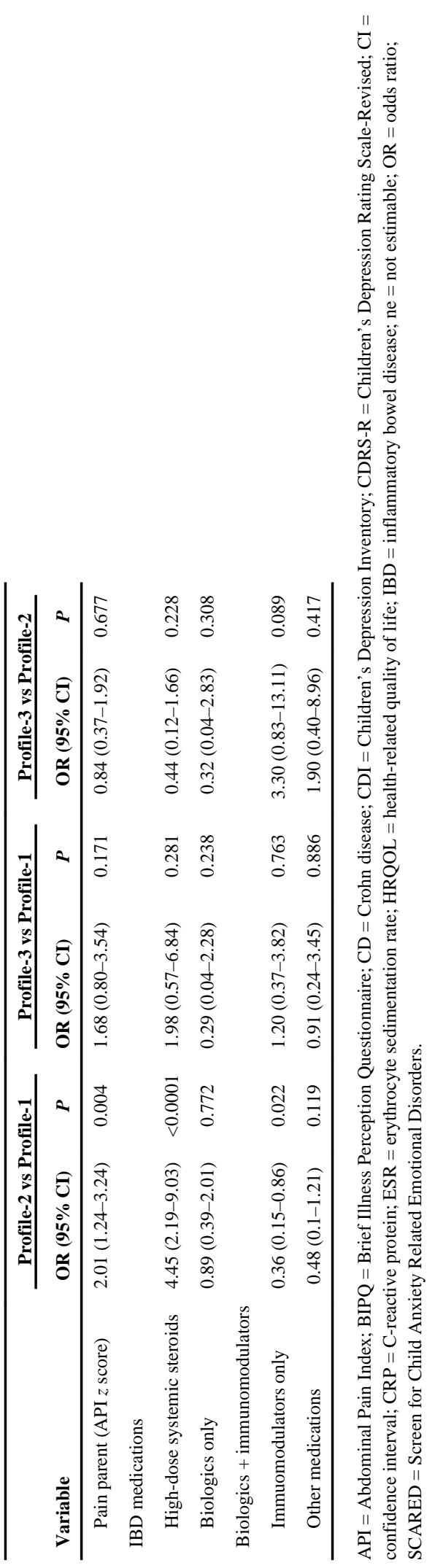

